\section{Ingeniería}

CONTENIDO

Artículos

Análisis numérico de un sistema de aire acondicionado empleando
mallas estructuradas y no estructurades A

Funciones de regresión para caudales extremos en la Vertiente Rojas, Nazareth; Aguilar, José Francisco; Solís, Hernán. Evaluación de daños por agresión ambiental en viviendas
de concreto reforzado. Solís, Rómel; Moreno, Eric; Jiménez, Felipe; Rosas, Victorino.

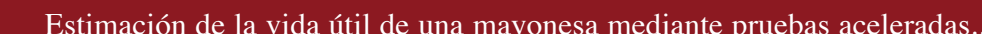
Estimación de la vida útil de una mayonesa
García, Claudia; Molina, Manuel.

Inestabilidad oscilatoria de tensión debido a los motores de inducción …….....................65-75 rde, Gustavo; Araya, Eddie.

Estabilidad y colapso de tensión en sistemas eléctricos Araya, Eddie.

Obtención de pulpa a la soda antraquinona de Tectona grandis creciendo en Costa Rica...........................

Protocolo evaluador para el manejo de desechos y la seguridad ocupacional
aplicado al laboratorio clínico del Hospital San Francisco de Asís de Grecia. Ruiz, Francisco.

9. Euler: su contex
Vargas, Celso.

10. Reserva de resistencia de edificios porticados de concreto armado disenaados conforme al ACI-318/ IBC 2006 ........ Análisis y comentarios

Herrera, Rodolfo.

breve semblanza.

Trabajos de Graduación 2007

Lista de proyectos de graduación de grado y posgrado

Normas

$\sum_{\text {EDTORAL }}$

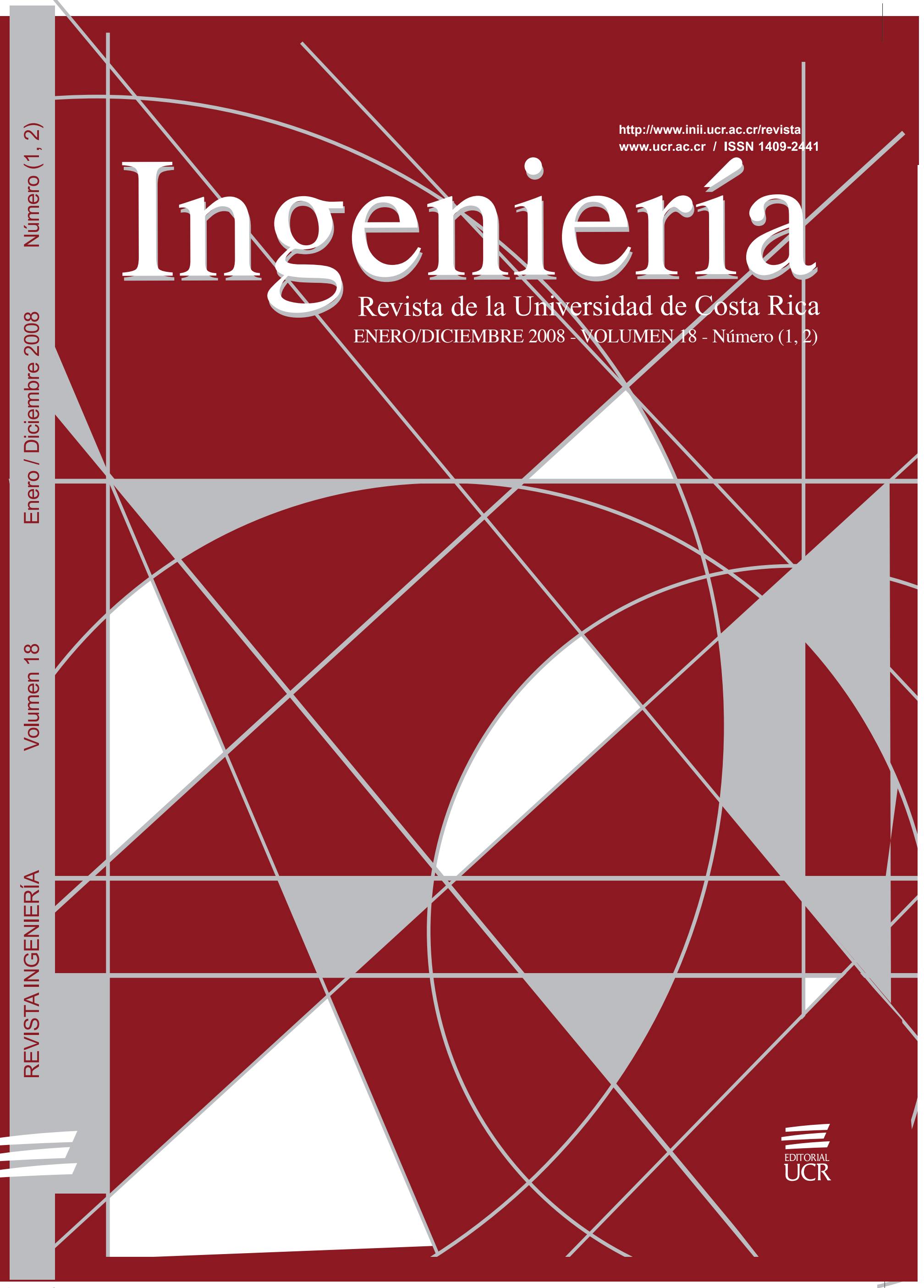




\title{
INESTABILIDAD OSCILATORIA DE TENSIÓN DEBIDO A LOS MOTORES DE INDUCCIÓN
}

\author{
Gustavo A. Valverde Mora \\ Eddie A. Araya Padilla
}

\begin{abstract}
Resumen
Dado que el desempeño de los motores de inducción está directamente relacionado a la estabilidad de tensión de la línea, en esta publicación los autores estudian su comportamiento bajo tal condición.

El método empleado fue analizar los puntos de equilibrio del motor. Para cada punto de operación, la estabilidad del motor fue evaluada con las curvas Potencia activa-Tensión (PV) y Potencia reactiva-Tensión (QV), sus autovalores y sus simulaciones dinámicas.

Del análisis llevado a cabo, se concluyó que tanto el punto de operación del motor, como la variación de la tensión de la línea, harán que se alcance el límite de estabilidad oscilatoria de tensión.
\end{abstract}

Palabras clave: inestabilidad de tensión, motores de inducción, autovalores, curvas PV y QV

\begin{abstract}
Given that performance of induction motor is directly related to line voltage stability; in this paper the authors study their behavior under such condition.

The method employed was to analyze equilibrium points of de motor. For each point of operation the motor stability was evaluated with Power-Voltage (PV) and Var- Voltage (QV) curves, their eigenvalues, and dynamic simulations.

From the analysis carried out, we concluded that both the point of operation of the motor and line voltage variation will make it reach the limit of oscillatory voltage stability.
\end{abstract}

Key words: voltage instability, induction motor, eigenvalues, PV and QV curves

Recibido: 07 de noviembre del 2008 • Aprobado: 10 de febrero del 2009

\section{INTRODUCCIÓN}

El creciente uso de motores compresores para aires acondicionados o refrigeración y los grandes motores de inducción en los centros de carga, han incentivado la investigación del fenómeno de inestabilidad de tensión de corto alcance, debido a que este tipo de cargas corresponden a componentes dinámicos de rápida acción, en presencia de una gran perturbación en el sistema. Estas cargas afectan significativamente la recuperación de la tensión, ante un cambio en sus condiciones operativas.
La inestabilidad de tensión es un proceso dinámico que involucra aspectos de las cargas, y es por ello que también se le conoce como inestabilidad de carga.

El fenómeno de inestabilidad de tensión abarca diferentes escalas de tiempo, clasificadas en inestabilidad de corto y gran alcance. El caso de inestabilidad de corto alcance se presenta en cargas dinámicas de rápida ac-ción como motores de inducción (MI), cargas controladas electrónicamente y convertidores HVDC (HVDC: High Voltage Direct Current, por sus siglas en inglés). 
En Van Cutsem \& Vournas (1998), se establece una subdivisión en las escalas de tiempo, donde se presentan los fenómenos de inestabilidad de tensión. En el caso del corto alcance, se tienen los siguientes mecanismos de inestabilidad:

- Pérdida de equilibrio post-perturbación, en dinámica de corto alcance.

- Escasez de atracción en relación con el equilibrio post-perturbación, en dinámica de corto alcance.

- Inestabilidad oscilatoria del equilibrio postperturbación.

Un ejemplo del primer mecanismo de inestabilidad se presenta cuando un MI se desacelera después de una perturbación. Una vez que el par mecánico es mayor que el eléctrico, el motor permanece en una condición de poca velocidad consumiendo altas corrientes, con la tensión en terminales muy baja. El segundo mecanismo de estabilidad se presenta cuando una vez eliminada la falla, el sistema se encuentra fuera de atracción de los puntos de equilibrio, dejando al sistema inestable. Finalmente, la inestabilidad oscilatoria corresponde a una condición menos común, para la cual el sistema se encuentra en el límite de inestabilidad, cuando los autovalores que definen el sistema se encuentran en el eje j $\omega$ del plano complejo. Mostrando así, un comportamiento oscilatorio, incluyendo la respuesta oscilatoria de la tensión. Este último mecanismo es el objetivo de estudio de esta publicación.

\section{CONDICIONES DE OPERACIÓN DE LOS MI}

Los MI demandan altas corrientes cuando arrancan o cuando son desacelerados por un cambio de carga o perturbación. Una vez que el motor opera a una velocidad cercana a la velocidad nominal, los valores de corriente disminuyen considerablemente y el motor alcanza un nuevo punto de equilibrio. Este comportamiento se muestra en la Figura 1, donde la corriente consumida y el par electromagnético desarrollado están en función de su deslizamiento.
Por otro lado, el par electromagnético del motor además de depender del deslizamiento $\mathrm{s}$, es proporcional al cuadrado de la tensión de suministro, en la forma:

$T e=f(V, s)$

En el caso de un decremento de la tensión de suministro producto de una falla, dará como resultado una re-ducción del par electromagnético en proporción cuadrática, desacelerando al motor (s aumenta), cuyo com-portamiento dependerá de la inercia de cada motor (Díaz \& Taylor, 2002).

En la Figura 1 se muestra cómo, a bajas velocidades, la corriente demandada por el motor es sumamente alta, debido al consumo de potencia reactiva. En este caso el factor de potencia es bajo y el par electromagnético no logra recuperar su estado inicial. Una vez que se elimina la falla, si el par eléctrico es mayor que el par mecánico, el motor reacelera a valores de velocidad cercanos a los nominales. En caso contrario, el motor se frenará demandando altas corrientes.

A partir de la Figura 2, se observa el comportamiento de dos modelos del par mecánico que representa la carga, en función del deslizamiento, as como el 1 par electromecánico desarrollado por el motor.

Los modelos de par mecánico en función del deslizamiento son:

$T_{m 1}(s)=T_{0}$

$T_{m 2}(s)=T_{2}(1-s)^{2}$

donde $T_{0}$ y $T_{2}$ son constantes que dependen del tipo de motor. Los puntos A, B, C y D muestran las intersec-ciones de las curvas, e indican si los puntos de operación en régimen permanente del motor, son estables o inestables identificados por las letras E e I, respectivamente.

Al analizar la curva del par mecánico constante, se observa que interseca la curva del par electromecánico en dos puntos, A y B. Si el motor se encuentra en el punto de operación $\mathrm{B}, \mathrm{y}$ ocurre un incremento en el desliza-miento, el par eléctrico se incrementa más que el par mecánico, provocando que el motor nuevamente aumen- 


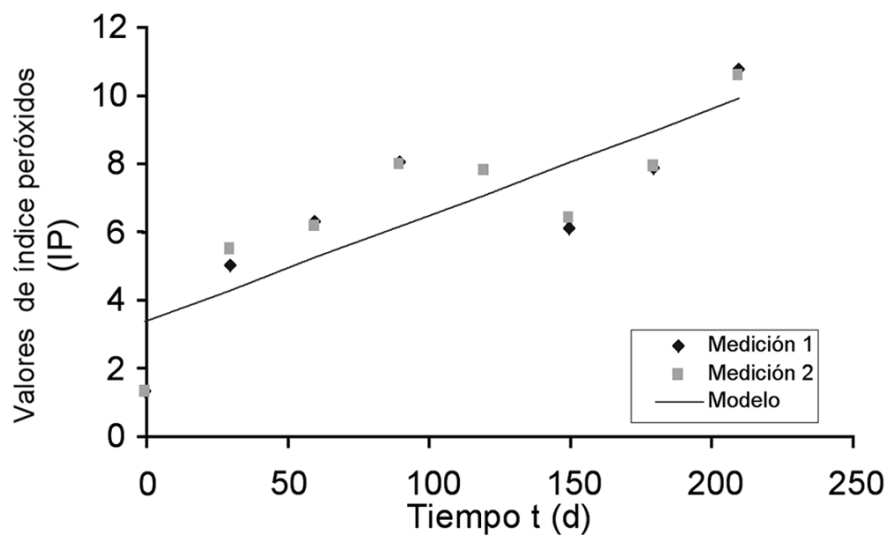

Figura 1. Par y corriente eléctrica de un motor de inducción en operación normal.

Fuente: (Valverde, 2005).

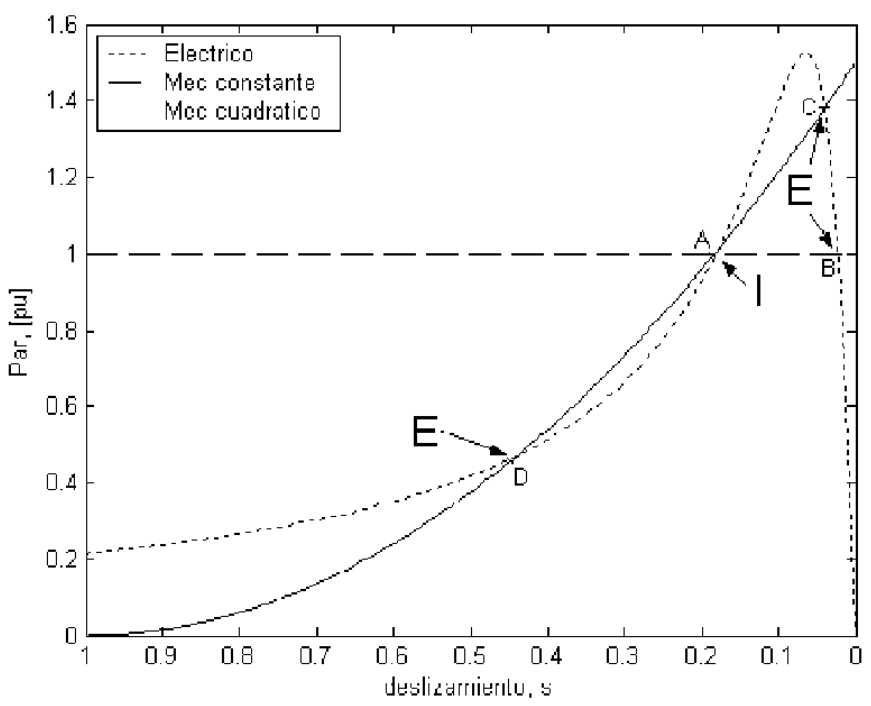

Figura 2. Par y corriente eléctrica de un motor de inducción en operación normal.

Fuente: (Valverde, 2005).

te su velocidad debido a la diferencia de los pares, y como consecuencia, el motor regresará al punto B. Nuevamente situados en el punto $\mathrm{B}$, un decremento del deslizamiento (aumento de velocidad) involucra que el par mecánico es ahora mayor que el eléctrico, y en esta situación el motor tiende a frenarse hasta que los pares nuevamente sean iguales, condición del punto B.
A dicho punto se le conoce entonces como punto de equilibrio estable E.

Por otro lado, si la condición de operación del motor es el punto A, una pequeña variación en la velocidad del motor conlleva a que se pierda la condición de equilibrio. Esto es, si el motor adquiere un pequeño incremento de velocidad, $s$ tiende a cero, el par eléctrico es mayor que 
el mecánico y el motor elevará su velocidad, alejándose así del punto de operación A. En el caso de un pequeño incremento del deslizamiento, el par mecánico es superior al eléctrico y el motor comenzará a frenarse. De lo anterior se concluye que A es un punto inestable I.

Para el modelo del par cuadrático, se puede realizar el mismo análisis con los mismos razonamientos anterio-res. Cabe destacar que el modelo utilizado en el ejemplo de la Figura 2 intersecó la curva del par eléctrico en tres puntos diferentes, aunque no siempre es así, ya que esto depende de los valores de en la ecuación (2).

En vista de lo anterior, la estabilidad depende del punto de operación en que se encuentra el motor, después de eliminada una falla. Para determinar su condición post-falla, se hace necesario un análisis más detallado.

\section{MODELOS DE CARGAS ASINCRÓNICAS}

Con el propósito de evaluar el efecto dinámico del motor de inducción sobre la estabilidad de tensión, en esta sección se presentan dos modelos típicos.

\subsection{Modelo simple del MI}

Este modelo se enfoca en la dinámica del deslizamiento, y se representa por la ecuación (Balanathan, 2002):

$\dot{s}=\frac{1}{2 H}\left(\frac{P_{m}}{1-s}-P\right)$

donde:

$P_{m}$ es la carga mecánica

$P \quad$ es la potencia eléctrica consumida

$s \quad$ el deslizamiento

$H$ es la constante de inercia del motor

En este modelo se desprecia la resistencia del estator y se supone que la reactancia de magnetización es muy grande (Balanathan, 2002), dando origen a un modelo constituido por la reactancia del estator Xs, la reactancia del rotor Xr y la resistencia del rotor $\mathrm{Rr}$.

Para facilitar el análisis, se obtiene el circuito equivalente visto desde el estator, como se muestra en la Figura 3, en donde la caída de tensión E en la resistencia del rotor, representa la tensión inducida en el circuito del rotor, y es conocida como la tensión detrás de la reactancia $\mathrm{Xs}+\mathrm{Xr}$.

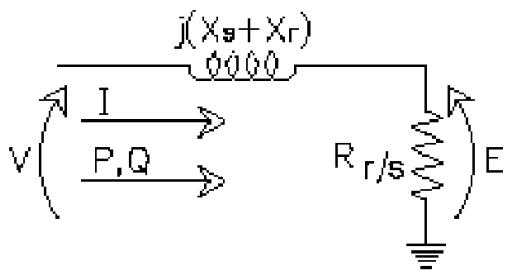

Figura 3. Circuito equivalente de MI.

Fuente: (Valverde, 2005).

Al efectuar una división de tensión, en el circuito de la Figura 3, se obtiene una expresión de la tensión E en función de la tensión aplicada y de los parámetros del motor, de la forma:

$$
E^{2}=\frac{V^{2}}{1+\left(\frac{s(X s+X r)}{R r}\right)^{2}}
$$

Así mismo, de la Figura 3 se tiene que:

$$
\bar{I}=\frac{\bar{V}}{\frac{R r}{s}+j(X s+X r)}
$$

A partir de la ecuación (6), se determinan las expresiones de las potencias activas y reactivas consumidas por el motor dadas por:

$$
P=\operatorname{Re}\left\{\overline{V I}^{*}\right\}=\frac{V^{2}}{(R r / s)^{2}+(X s+X r)^{2}} \frac{R r}{s}
$$

$$
Q=\operatorname{Im}\left\{\overline{V I}^{*}\right\}=\frac{V^{2}}{(R r / s)^{2}+(X s+X r)^{2}}(X s+X r)
$$




\subsection{Modelo detallado del MI}

En este caso, el modelo detallado del motor incorpora la reactancia de magnetización, tal como se muestra en la Figura 4.

A partir de la Figura 4, se obtienen los circuitos equivalentes de la Figura 5 para un mejor análisis.

De los cuales:

$$
\begin{aligned}
& R_{1}+j X_{1}=\frac{j X_{m}\left(R_{s}+j X_{s}\right)}{R_{s}+j\left(X_{s}+X_{m}\right)} \\
& V_{1}=\frac{X_{m} V}{\sqrt{R_{s}^{2}+\left(X_{s}+X_{m}\right)^{2}}}
\end{aligned}
$$

La corriente que consume el motor se determina con ayuda de la Figura 5 y se expresa como:

$\bar{I}=\frac{\bar{V}}{\left(R_{s}+R_{e}\right)+j\left(X_{s}+X_{e}\right)}$

donde Re y Xe son:

$$
R_{e}+j X_{e}=\frac{j X_{m}\left(\frac{R_{r}}{s}+j X_{r}\right)}{\frac{R_{r}}{s}+j\left(X_{r}+X_{m}\right)}
$$

Finalmente, utilizando la ecuación (11), se determinan las expresiones de las potencias activas y reactivas consumidas por el motor:

$$
\begin{aligned}
& P=\frac{\left(R_{s}+R_{e}\right) V^{2}}{\left(R_{s}+R_{e}\right)^{2}+\left(X_{s}+X_{e}\right)^{2}} \\
& Q=\frac{\left(X_{s}+X_{e}\right) V^{2}}{\left(R_{s}+R_{e}\right)^{2}+\left(X_{s}+X_{e}\right)^{2}}
\end{aligned}
$$

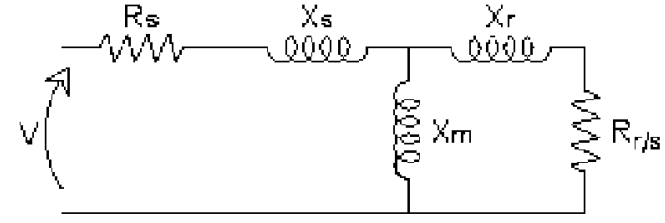

Figura 4. Circuito equivalente del MI. Fuente: (Valverde, 2005).
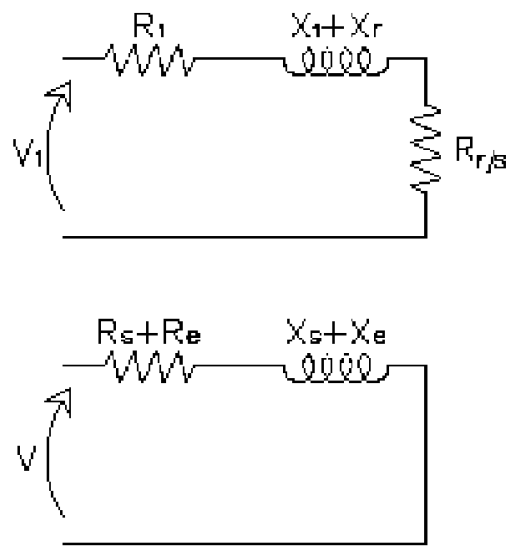

Figura 5. Circuito equivalente del MI visto desde diferentes terminales.

Fuente: (Valverde, 2005).

\section{ANÁLISIS DE LA INESTABILIDAD OSCILATORIA DE TENSIÓN}

En el estudio de la estabilidad oscilatoria de tensión, es necesario conocer el comportamiento pre-falla del sistema para determinar las condiciones iniciales de las variables de interés. Luego, mediante algún cambio en los parámetros del sistema, se localizan los puntos de equilibrio.

Un estudio preliminar en régimen permanente, permite encontrar las condiciones iniciales para realizar posteriormente las simulaciones en el dominio del tiempo. De esta forma, el cambio en el valor de alguno de los parámetros permite ubicar al motor en un punto inestable y de esta forma, observar su comportamiento.

\subsection{Análisis del modelo simple del MI}

Con el propósito de establecer cómo los motores de inducción afectan la estabilidad de 
tensión, se estudia en primera instancia el modelo del motor de inducción expuesto en la sección 3.1.

En este caso, el par mecánico puede ser representado por:

$T_{m}=T_{o}(1-s)^{n}$

donde

$T_{0}$ es un valor constante del par mecánico

$\mathrm{n}$ es un parámetro útil para representar diferentes modelos de carga mecánica

Por otro lado, se sabe que la carga mecánica puede ser expresada como:

$$
P_{m}=T_{m}(1-s)
$$

De modo que sustituyendo (15) en (16) se obtiene:

$$
\frac{P_{m}}{1-s}=T_{o}(1-s)^{n}
$$

Finalmente, al sustituir (17) en (4), se determina la ecuación dinámica del motor de inducción:

$$
\dot{s}=\frac{1}{2 H}\left(T_{o}(1-s)^{n}-P\right)
$$

donde la potencia activa corresponde a la ecuación (7).

Ahora bien, para establecer el comportamiento del motor en diferentes puntos de operación, se asume que es-te se encuentra conectado a una barra de tensión constante $\mathrm{V}$, y se realiza el estudio en régimen permanente haciendo en la relación (18). En este caso se obtiene la expresión:

$$
T_{o}(1-s)^{n}=\frac{V^{2}}{(R r / s)^{2}+\left(X_{s}+X_{r}\right)^{2}} \cdot \frac{R r}{s}
$$

A partir de (19), para una condición dada de $\mathrm{s}$, se determinan los valores de $\mathrm{V}$ y $\mathrm{P}$, conociendo los valores de las constantes $\mathrm{n}$ y. Con estos datos es posible obtener la curva PV del motor. La Figura 6 muestra las regiones de equilibrio del motor para un par mecánico cuadrático $(n=2)$, con la constante igual a 1.2 p.u.

Por otro lado, mediante el cálculo de los autovalores para los puntos de operación, es posible conocer la esta-bilidad del sistema, cuando se varía la potencia del motor. La ecuación dinámica del motor dada por (18) de-be ser linealizada alrededor del punto de operación, para conocer su condición de estabilidad.

Como lo muestra la Figura 7, el único autovalor real del modelo de motor, cambia según el punto de operación. Para valores de potencia cercanos a 1,2 p.u el sistema es estable, lo cual corresponde a velocidades cercanas a las de plena carga. Luego, el sistema posee una rama de equilibrio inestable y finalmente, una tercera rama de puntos de equilibrio estables, para valores de potencia menores de 0,5 p.u.

A partir de la Figura 7, el motor puede encontrarse en una condición estable o inestable. De modo que, para complementar el análisis de estas condiciones se realiza la simulación del motor de inducción, sujeto a cambios en la tensión en sus terminales.

Teniendo en cuenta que la rama de equilibrio cercana a 1,2 p.u es estable, se realiza una simulación variando la tensión de terminal a un valor menor, pero que esté dentro de la región estable. Se varía la tensión de 1.0 p.u hasta 0,8 p.u a los dos segundos de simulación, tal como se muestra en la Figura 8, donde se presenta el comportamiento de la potencia activa consumida por el motor. Luego del cambio en la tensión, la potencia cae pero regresa al valor pre-falla porque se sitúa en un punto de operación todavía estable. La diferencia se debe a que el motor trabaja con un nuevo valor de deslizamiento, y la potencia reactiva $\mathrm{Q}$ consumida por el motor también aumenta.

En su defecto, si se realiza una nueva simulación pero en un punto inestable de la curva $\mathrm{PV}$, el cambio de la tensión en terminales hará que el motor no posea la suficiente cantidad de potencia reactiva $\mathrm{Q}$ para reestablecer la 


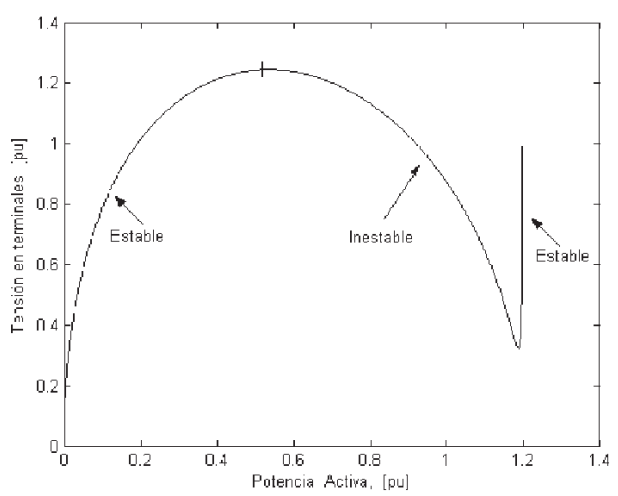

Figura 6. Curva PV del modelo de M.I simple para .

$$
T_{0}=1.2 p . u \text {. }
$$

Fuente: (Valverde, 2005).

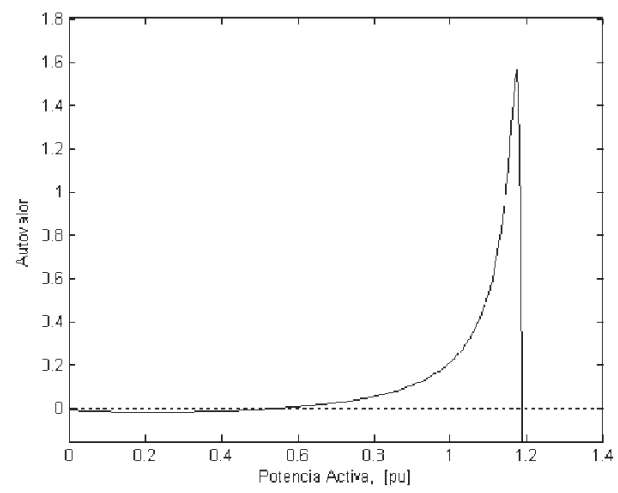

Figura 7. Autovalor real al variar la potencia activa $P$. Fuente: (Valverde, 2005).

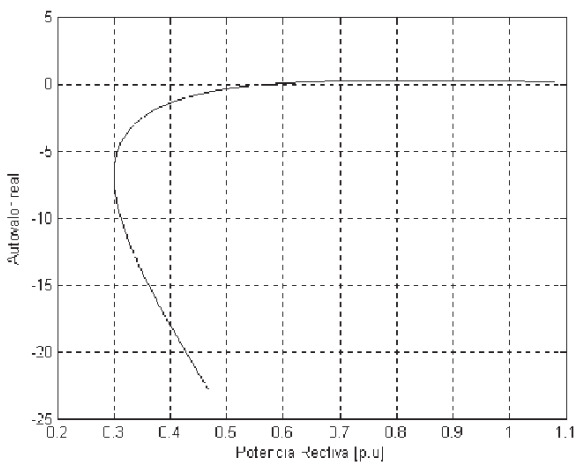

Figura 8. Simulación caso estable. Fuente: (Valverde, 2005). tensión, provocando que el motor se detenga. Este comportamiento se debe a que el par eléctrico es menor que el par mecánico. Dicho comportamiento inestable se muestra en la Figura 9, donde la potencia activa $\mathrm{P}$ del motor disminuye, aun cuando en los primeros instantes trata de recuperar su valor inicial, pero al no recuperar la tensión en terminales, la potencia activa $\mathrm{P}$ tiende a caer a los pocos segundos.

Este sencillo análisis demuestra cómo las condiciones iniciales de operación afectan el comportamiento post-falla y permite observar la rapidez en la dinámica del motor.

\subsection{Análisis del modelo detallado del MI}

Para ampliar el análisis anterior, se usará en esta sección un modelo más complejo para obtener otros resultados. El modelo de motor usado para el nuevo análisis de estabilidad se estudió en la sección 3.2, junto con la ecuación dinámica del motor:

$2 H \dot{s}=T m-T e$

donde Tm es el modelo del par mecánico y Te es el par eléctrico del motor que depende del deslizamiento y la tensión en terminales del motor, $\mathrm{Te}=\mathrm{f}(\mathrm{V}, \mathrm{s})$.

Con el fin de realizar el diagrama de bifurcación en régimen permanente, , se grafican los puntos de equilibrio del motor, donde:

$T m=T e$

Para una condición dada de deslizamiento, se calcula la tensión en terminales del motor conociendo el par mecánico constante o cuadrático. El cálculo de $\mathrm{P}$ y $\mathrm{Q}$ se obtiene sustituyendo el valor de $\mathrm{s}$ y el respectivo valor de $\mathrm{V}$, en las ecuaciones, para cada punto de operación.

Las curvas PV y QV se realizaron para el par mecánico constante y cuadrático. En el caso del par cuadrático, los valores que se especifican en las gráficas corresponden al parámetro constante de la ecuación (3). 
A partir de las Figuras 10 a 13 se notan las diferencias al usar distintos modelos de par mecánico y cómo se desplaza la curva al aumentar las constantes del par.

En el caso de la Figura 10, se muestran las curvas PV de un motor de inducción con carga mecánica constante. La variación de la carga se muestra en la figura al apreciarse un desplazamiento hacia la derecha al aumentar el valor de $T_{m}$.

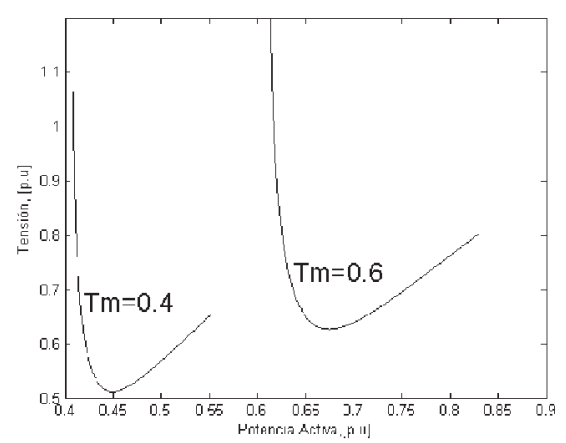

Figura 10. Curvas PV de motor de inducción con $T_{m}$ constante.

Fuente: (Valverde, 2005).

Para efectos de observar el comportamiento de la tensión, al aumentar la potencia reactiva, se grafican las curvas QV para dos valores diferentes de $T_{m}$. Para un mismo valor de tensión, la potencia reactiva es mayor si la carga mecánica aumenta, tal como se muestra en la Figura 11.

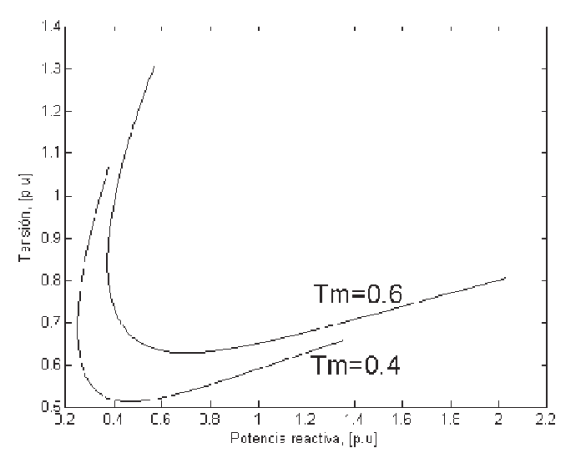

Figura 11. Curvas QV de motor de inducción con $T_{m}$ constante.

Fuente: (Valverde, 2005).
Ahora bien, como la carga mecánica de un motor de inducción no siempre es constante, el modelo de par cuadrático representa con mayor precisión la carga mecánica de motores. Nuevamente se grafican las curvas PV y QV para diferentes valores de $T_{2}$, para apreciar el desplazamiento de las curvas al aumentar la carga mecánica.

La Figura 12 presenta la curva PV, mediante la variación de la potencia activa $\mathrm{P}$, para dos valores del parámetro $T_{2}$, del modelo cuadrático del par mecánico.

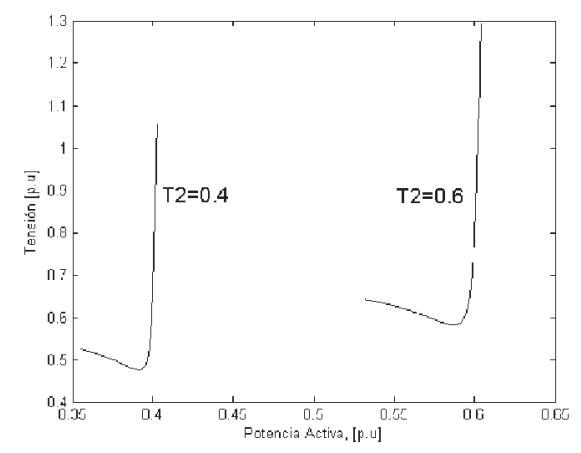

Figura 12. Curvas PV de motor de inducción con $T_{m}$ cuadrático.

Fuente: (Valverde, 2005).

De forma similar, la Figura 13 muestra la curva $\mathrm{QV}$, mediante la variación de la potencia reactiva $\mathrm{Q}$, para dos valores del parámetro , del modelo cuadrático del par mecánico.

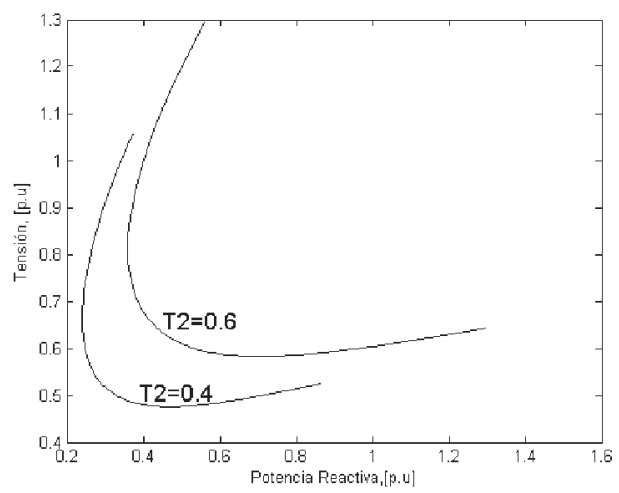

Figura 13. Curvas QV de motor de inducción con $T_{m}$ cuadrático. Fuente: (Valverde, 2005). 
Ahora bien, con el propósito de evaluar más detalladamente el caso del motor con par mecánico cuadrático, $T_{2}=0.5$, se realiza un análisis de estabilidad para conocer cuáles puntos de equilibrio son estables e inestables. Estas regiones de estabilidad se muestran en la Figura 14. Una vez conocido el autovalor, se puede determinar la región de puntos de equilibrio donde el motor es estable, E, o inestable, I.

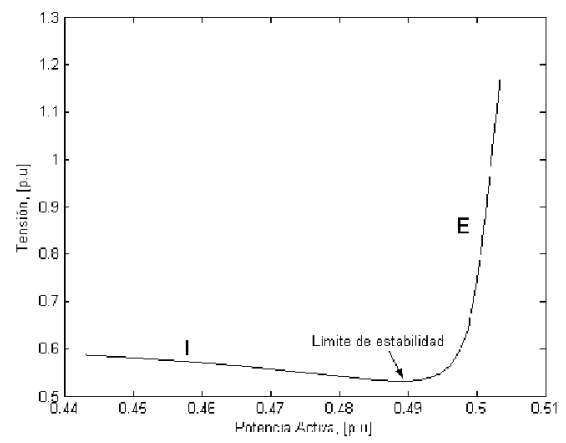

Figura 14. Curva PV del motor de inducción para $T_{2}=0.5$ p.u.

Fuente: (Valverde, 2005).

En este caso, cuando $T_{2}=0.5$ p.u., el autovalor en función de la potencia activa $\mathrm{P}$ se muestra en la Figura 15.

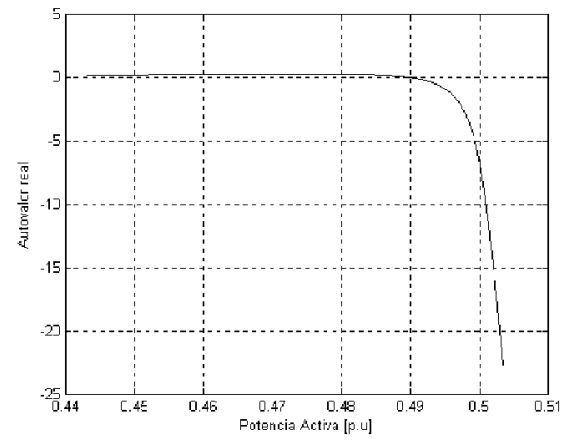

Figura 15. Comportamiento del autovalor al variar la potencia activa.

Fuente: (Valverde, 2005).

De forma similar, la curva QV del motor, para un par mecánico cuadrático con $T_{2}=0,5$ p.u., se muestra en la Figura 16. Una vez conocido el autovalor, se puede determinar la región de puntos de equilibrio donde el motor es estable, E, o inestable, I.

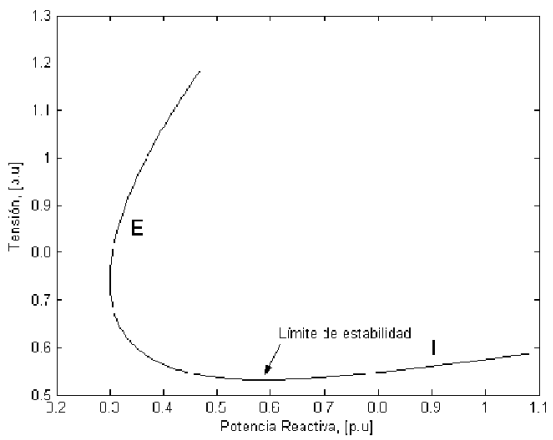

Figura 16. Curva QV del motor de inducción para $T_{2}=0.5$ p.u.

Fuente: (Valverde, 2005).

Para determinar la estabilidad de los puntos de equilibrio, la Figura 17 presenta cómo cambia el autovalor en función de la potencia reactiva $\mathrm{Q}$.

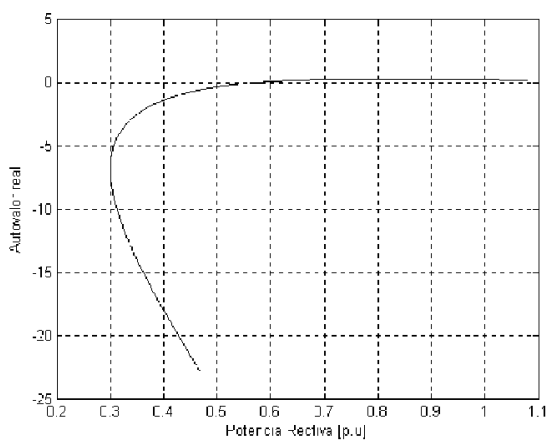

Figura 17. Comportamiento del autovalor al variar la potencia reactiva.

Fuente: (Valverde, 2005).

Para visualizar el comportamiento dinámico del motor de inducción, se realizan las simulaciones para cambios en la tensión terminal. En el primer caso, la variación de la tensión se produce de tal forma que el sistema alcance un punto de operación estable, y en el segundo caso, la variación de la tensión lleva al motor a un punto de la región inestable de las Figuras 14 y 16.

La Figura 18 muestra el comportamiento del motor cuando, después de una perturbación en la 
tensión, alcanza un punto de operación estable. Despuésdeltransitorio,elmotoraproximadamente consume 0,5 p.u. de potencia activa. La potencia activa post-falla es prácticamente igual a la potencia pre-falla, debido a que el cambio no se presentó en la carga mecánica del motor, sino en la tensión de suministro.

Al comparar el comportamiento del motor durante la simulación en el dominio del tiempo, con la curva $P V$ de la Figura 14, se comprueba que esta condición operativa se encuentra en la rama estable del diagrama.

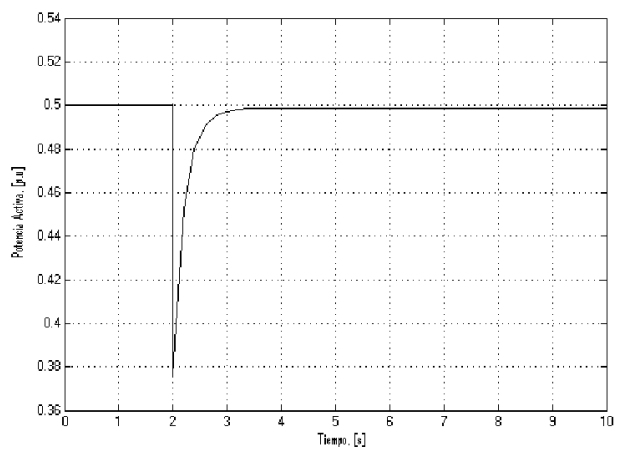

Figura 18. Simulación de P ante un cambio en tensión terminal, caso estable.

Fuente: (Valverde, 2005).

Con respecto a la potencia reactiva consumida por el motor, la Figura 19 muestra la simulación donde el sistema alcanza un punto estable, luego de una perturbación en la tensión. En este caso, el sistema se estabiliza aproximadamente en 0,315 p.u de la potencia reactiva consumida por el motor. Al comparar la curva $Q V$ con la Figura 16 , se nota que este comportamiento pertenece a la rama estable del diagrama.

Del análisis anterior, la potencia reactiva aumentó al existir una disminución de la tensión en terminales del motor, lo que implica una reducción del par eléctrico y por tanto, este incremento de potencia reactiva, corresponde a la recuperación del par eléctrico pre-falla, debido a la caída de tensión.

Como segundo caso de análisis, en las Figuras 20 y 21, se ha simulado cuando el motor trabaja en un punto de operación inestable. Estos puntos de operación corresponden a valores

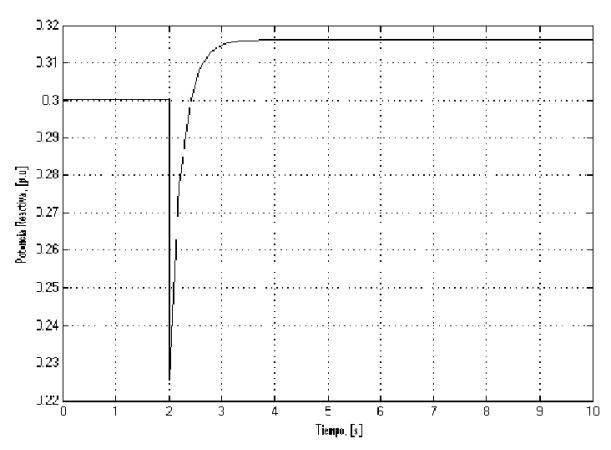

Figura 19. Simulación de Q ante un cambio en tensión terminal, caso estable.

Fuente: (Valverde, 2005).

menores a 0,4 p.u en potencia activa y mayores a 0,8 p.u en potencia reactiva, que corrobora los puntos inestables en las curvas PV (Figura 14) y QV (Figura16) del motor de inducción. En este análisis, el motor se encuentra consumiendo gran cantidad de potencia reactiva y se frena debido a que no es capaz de satisfacer la carga mecánica. $\mathrm{Su}$ factor de potencia es sumamente bajo, tendiendo a valores similares a las condiciones de arranque.

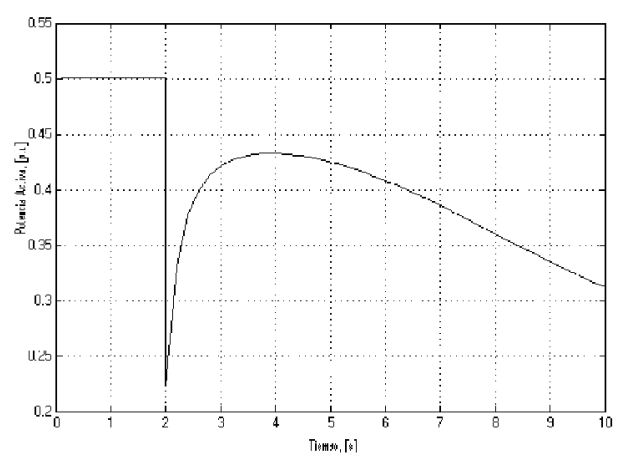

Figura 20. Simulación de $\mathrm{P}$ ante un cambio en tensión terminal, caso inestable.

Fuente: (Valverde, 2005).

A partir de la simulación, la Figura 20 muestra cómo la potencia activa del motor disminuye a los dos segundos, instante en que se inicia una recuperación en la caída de tensión. El motor intenta recuperar la potencia activa perdida, pero al encontrarse en un punto de operación 
inestable, el par mecánico es mayor al eléctrico y el motor se detiene a los pocos segundos. En esta condición, la Figura 21 muestra cómo la potencia reactiva consumida por el motor se incrementa considerablemente.

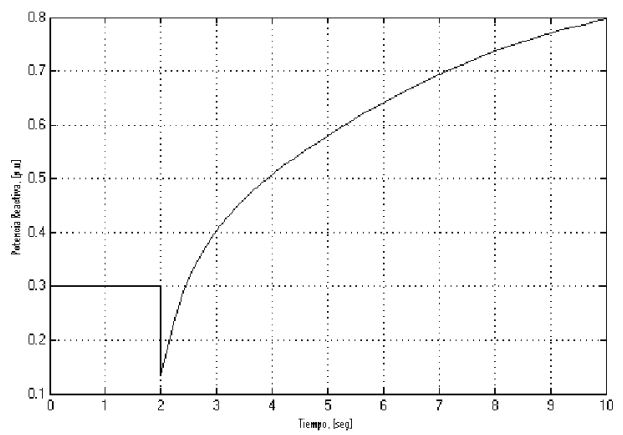

Figura 21. Simulación de Q ante un cambio en tensión terminal, caso inestable.

Fuente: (Valverde, 2005).

\section{CONCLUSIONES}

1. $\quad$ El motor de inducción, puede alcanzar el límite de estabilidad oscilatoria de tensión, dependiendo de su punto de operación, así como de la variación de la tensión de la línea.

2. Ciertas variaciones en la magnitud de de la tensión terminal de los motores de inducción, conllevan a que este absorba cantidades adicionales de potencia reactiva, similares a la condición de arranque.

3. El consume de altas corrientes reactivas por parte de los motores de inducción, contribuyen a la inestabilidad oscilatoria de tensión.

\section{REFERENCIAS BIBLIOGRÁFICAS}

Balanathan, R., Pahalawaththa N. \& Annakkage, U. (2002). Modeling induction motor loads for voltage stability analysis. Electrical Power \& Energy System, 24(12) 469-480.

Díaz, J \& Taylor, C. (2002). Understanding and solving short-term voltage stability problems. IEEE Transaction on Power Systems. July, 3(8), 745-752.

Valverde, G. (2005). Análisis de la estabilidad oscilatoria de tensión. Proyecto Eléctrico. Escuela de Ingeniería Eléctrica. Universidad de Costa Rica. San José, Costa Rica.

Van Cutsem, T. \& Vournas, C. (1998). Voltage stability of electric power systems (pp. 265266). Kluwer Academic Publishers.

\section{NOMENCLATURA SOBRE LOS AUTORES}

\section{Gustavo A. Valverde Mora}

Profesor Interino.Departamento de Máquinas Eléctricas y Sistemas de Potencia, Escuela de Ingeniería Eléctrica, Universidad de Costa Rica.

Su área de interés son los sistemas eléctricos de potencia de alta tensión.

Correo electrónico: gvalverde@eie.ucr.ac.cr

\section{Eddie A. Araya Padilla}

Profesor Catedrático.

Departamento de Maquinas Eléctricas y Sistemas de Potencia, Escuela de Ingeniería Eléctrica, Universidad de Costa Rica.

Sus áreas de interés son la dinámica no lineal, dispositivos FACTS y la generación eólica.

Correo electrónico: earaya@eie.ucr.ac.cr 
